\title{
An Unusual Presentation of Metastatic Breast Carcinoma
}

\author{
Maria Mendonça-Sanches ${ }^{1}$, Inês Rolim², José Cabeçadas², Margarida Rafael ${ }^{3}$ \\ ${ }^{1}$ Dermatology Department, Hospital de Santa Maria, Lisbon, Portugal \\ 2Pathology Department, Instituto Português de Oncologia de Lisboa Francisco Gentil, Lisbon, Portugal \\ ${ }^{3}$ Dermatology Department, Instituto Português de Oncologia de Lisboa Francisco Gentil, Lisbon, Portugal
}

Received: 16/03/2019

Accepted: 18/03/2019

Published: 08/04/2019

How to cite this article: Mendoça-Sanches M, Rolim I, Cabedaças J, Rafael M. An unusual presentation of metastatic breast carcinoma. EJCRIM 2019;6: doi:10.12890/2019_001089.

Conflicts of Interests: The Authors declare that there are no competing interests.

This article is licensed under a Commons Attribution Non-Commercial 4.0 License

\section{ABSTRACT}

Cutaneous metastasis from a carcinoma is a relatively uncommon phenomenon. Prompt diagnosis is crucial, as it will have future implications, particularly regarding prognosis and treatment. Skin metastases can be suspected and recognized earlier through physical examination than metastases in other organs or systems. However, they can be a diagnostic challenge due to the variable clinical presentation. This case highlights the importance of having a high index of suspicion for cutaneous metastases, especially in patients with a previous history of cancer.

\section{LEARNING POINTS}

- Cutaneous metastases occur in approximately $0.7-10 \%$ of patients with invasive carcinomas and, when present, usually indicate an unfavourable outcome.

- Clinically, cutaneous metastasis can pose a diagnostic challenge and a high index of suspicion is mandatory for a prompt diagnosis.

- Skin biopsy of such lesions is important to confirm the diagnosis and, in the right clinical scenario, they can provide information on the primary origin of the tumour.

\section{KEYWORDS}

Cutaneous metastases, breast carcinoma, invasive carcinomas, skin biopsy

\section{INTRODUCTION}

Cutaneous metastases are defined as neoplastic lesions affecting the dermis or the subcutaneous tissue that originate from another primary malignancy. They are a relatively uncommon phenomenon with a reported incidence in the literature ranging from $0.7 \%$ to $10 \%{ }^{[1,2]}$. Once diagnosed, cutaneous metastases indicate a poor clinical outcome as they are related to a higher stage, later course and/or an unsuccessful response to treatment ${ }^{[1-4]}$.

Compared with metastases in other organs or systems, skin metastases can be suspected and promptly recognized by a clinician through physical examination ${ }^{[4]}$. However, the clinical presentation can be a diagnostic challenge as the lesions are frequently asymptomatic and may present in variable forms such as papules, nodules, plaques, or inflammatory and bullous lesions ${ }^{[1,5-7]}$. Skin biopsy evaluation of such lesions is fundamental as it can provide information on the primary origin of the tumour ${ }^{[4,8]}$.

\section{CASE REPORT}

A 52-year-old Caucasian woman was seen in our outpatient clinic for the recent appearance over the previous 6 months of numerous asymptomatic skin lesions on the neck, trunk and proximal regions of the upper and lower limbs. Her medical history included a diagnosis of right-sided invasive lobular breast carcinoma (cT3N+, oestrogen and progesterone receptor positive, ERBB2 negative) 9 years earlier. She 
had undergone neoadjuvant chemotherapy and a right radical mastectomy followed by local radiotherapy, adjuvant chemotherapy and 5 years of endocrine therapy with tamoxifen.

In the past 2 years, stage IV breast cancer with multifocal metastatic bone disease had been documented. Letrozole therapy was given initially, with partial response, but during the previous year there had been continuing on-going symptomatic bone involvement. The patient had declined further medical treatment and initiated alternative therapies, namely ozonotherapy. On physical examination, multiple skincoloured to brown papules were seen on the neck, trunk, arms and thighs (Fig. 1). A punch biopsy was performed and confirmed the diagnosis of cutaneous infiltration by neoplastic cells, compatible with metastatic lobular carcinoma of the breast (Fig. 2).

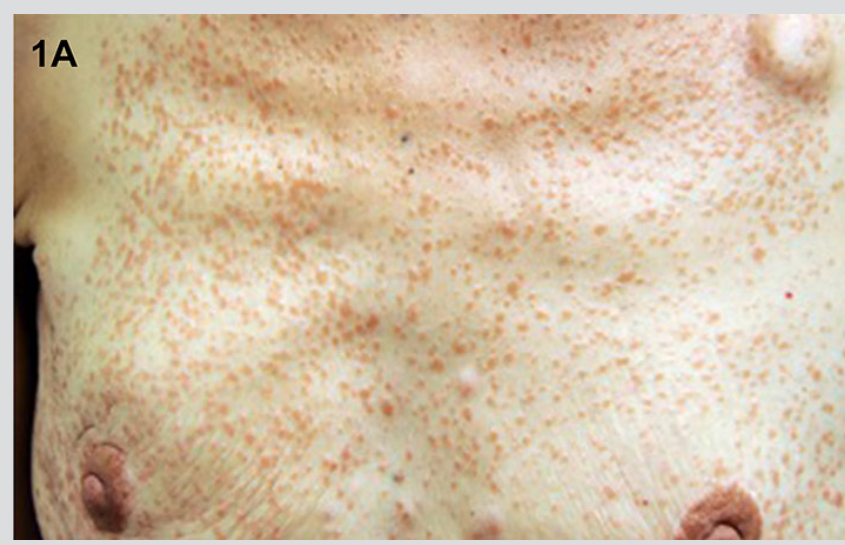

\section{B}

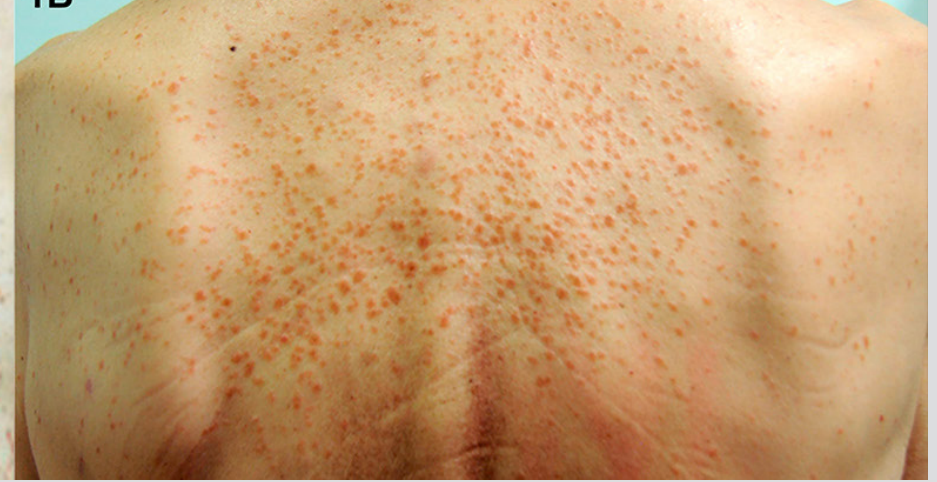

Figure 1. Multiple skin-coloured to brown papules on (A) the upper chest and (B) the upper back

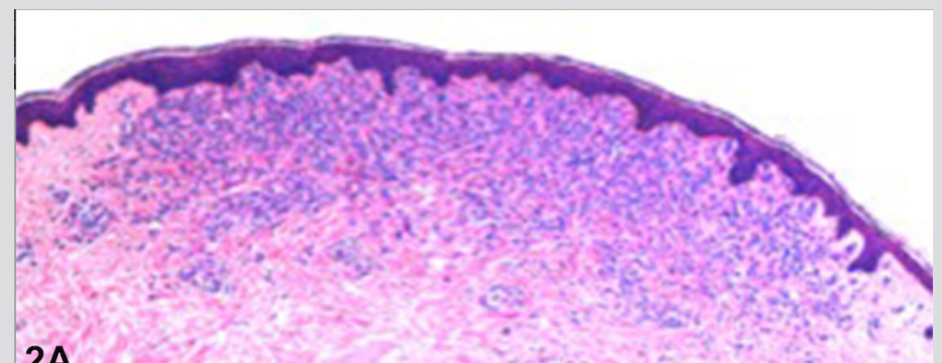

$2 A$
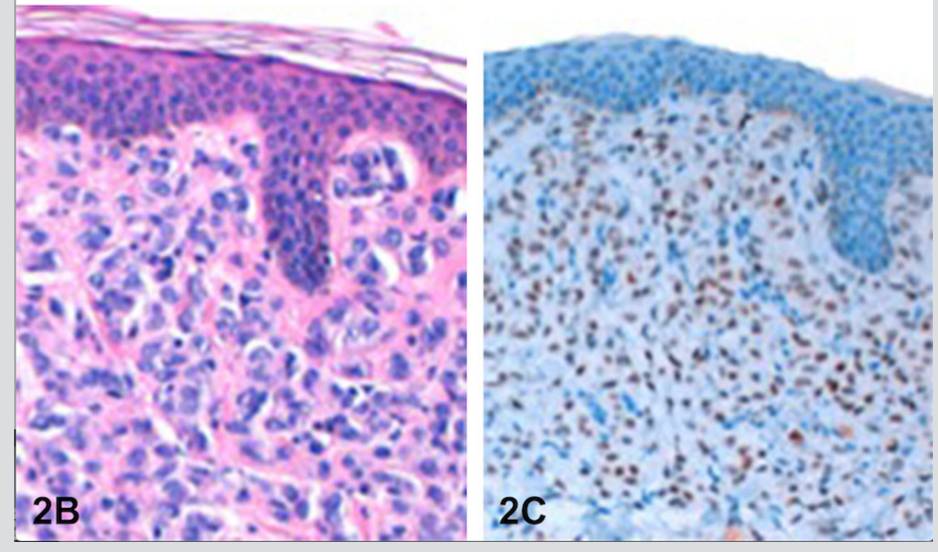

Figure 2. (A) Epithelial cells infiltrate the superficial and reticular dermis. (B) The tumour cells show moderate nuclear atypia and scant eosinophilic cytoplasm. (C) Positive nuclear staining for oestrogen receptors in the neoplastic cells

\section{DISCUSSION}

Cutaneous metastases occur in approximately $0.7-10 \%$ of patients with invasive carcinomas. Although uncommon, and comprising approximately $2 \%$ of all skin tumours, when present they usually predict a poor prognosis ${ }^{[5]}$. These skin manifestations are usually seen in patients with advanced disease, but they can be the presenting sign of a neoplastic disorder in $12 \%$ of the patients ${ }^{[7]}$. According to the literature, skin metastasis can be the first sign of metastatic involvement, particularly in breast cancer (in $23.9 \%$ of patients) and melanoma ${ }^{[1,8]}$. 
El Khoury et al. reported that in these patients, the most common primary origin was breast carcinoma in women and laryngeal carcinoma in men ${ }^{[9]}$.

With an aging population and increases in the average life expectancy and the incidence of cancer, the global burden of cancer is expected to grow. It is therefore important for clinicians and pathologists to be aware of the clinical spectrum of cutaneous metastases, and to have a high index of suspicion for early diagnosis and prompt management ${ }^{[5,7]}$. Gan et al. reported that in a cohort of 35 patients with confirmed cutaneous metastases, the diagnosis was suspected in $72 \%(n=25)$, based on clinical features $(46 \%, n=16)$ or the presence of active metastatic disease elsewhere $(26 \%, n=9)^{[5]}$. Accurate recognition of cutaneous metastases on biopsy examination is also crucial. In cases of an unknown primary tumour, histological and immunohistochemical evaluation is important to guide clinical and imaging investigations. Moreover, in cases of a previously documented tumour, cutaneous metastases may upstage the tumour and change the prognosis ${ }^{[4]}$.

In summary, the presented clinical case highlights the importance of early assessment of patients with cutaneous lesions, especially if there is a previous history of cancer, regardless of stage of treatment. This may lead to the prompt diagnosis of cutaneous metastases, which will have future implications, particularly regarding clinical outcome and treatment options.

\section{REFERENCES}

1. Hu SC, Chen GS, Lu YW, Wu CS, Lan CC. Cutaneous metastases from different internal malignancies: a clinical and prognostic appraisal. J Eur Acad Dermatol Venereol 2008;22:735-740.

2. Schwartz RA. Cutaneous metastatic disease. J Am Acad Dermatol 1995;33:161-182.

3. Lookingbill DP, Spangler N, Helm KF. Cutaneous metastases in patients with metastatic carcinoma: a retrospective study of 4020 patients. J Am Acad Dermatol 1993;29:228236.

4. Chopra R, Chhabra S, Samra SG, Thami GP, Punia RP, Mohan H. Cutaneous metastases of internal malignancies: a clinicopathologic study. Indian J Dermatol Venereol Leprol 2010;76:125-131.

5. Gan EY, Chio MT, Tan WP. A retrospective review of cutaneous metastases at the National Skin Centre Singapore. Australas J Dermatol 2015;56:1-6.

6. Saeed S, Keehn CA, Morgan MB. Cutaneous metastasis: a clinical, pathological, and immunohistochemical appraisal. J Cutan Pathol 2004;31:419-430.

7. Sariya D, Ruth K, Adams-McDonnell R, Cusack C, Xu X, Elenitsas R, et al. Clinicopathologic correlation of cutaneous metastases: experience from a cancer center. Arch Dermatol 2007;143:613-620.

8. Krathen RA, Orengo IF, Rosen T. Cutaneous metastasis: a meta-analysis of data. South Med J 2003;96:164-167.

9. El Khoury J, Khalifeh I, Kibbi AG, Abbas O. Cutaneous metastasis: clinicopathological study of 72 patients from a tertiary care center in Lebanon. Int J Dermatol 2014;53:147158. 